\title{
Incidencia lesiva en jugadores españoles de élite de balonmano playa Incidence of injury in elite Spanish beach handball players
}

*Alfonso Penichet-Tomás, **Manuel Ortega Becerra, *José Manuel Jiménez-Olmedo, *Basilio Pueo, *José Julio Espina Agulló *Universidad de Alicante (España), **Universidad Pablo Olavide (España)

Resumen: El objetivo del estudio fue determinar las lesiones más frecuentes en jugadores españoles de élite de balonmano playa. Ochenta deportistas de élite de, procedentes de veinte diferentes clubs participantes en el Arena Handball Tour de la Real Federación Española de Balonmano, participaron voluntariamente en el estudio. Para el tratamiento de los datos se realizó el test Chi-cuadrado ( $\mathrm{x}^{2}$ ) para pruebas no paramétricas. El análisis estadístico de los resultados $(p<.05)$ indicó que la región corporal en la que se produjeron más lesiones fueron las extremidades inferiores (54.2\%), más concretamente los músculos isquiotibiales (23.7\%) y el diagnóstico más repetido esguince (28.8\%). El tipo de lesión más reiterada fue la nueva lesión (66.1\%), siendo el impacto (57.6\%) el modo más frecuente. También se obtuvieron datos acerca del momento en el que se produjeron más lesiones, la competición (61.0\%). El 26.3\% sufre dolor crónico a causa de una vieja lesión. Este estudio ofrece información determinante a especialistas para la prevención de lesiones en la preparación de jugadores de élite.

Palabras clave: lesión, balonmano playa, cuestionario, arena, rendimiento.

Abstract: The aim of the study was to determine the most frequent injuries in elite Spanish beach handball players. Eighty elite athletes from twenty different clubs participating in the Arena Handball Tour of the Royal Spanish Handball Federation voluntarily participated in the study. For the treatment of the data, the Chi-square test was performed $\left(\mathrm{x}^{2}\right)$ for non-parametric tests. The statistical analysis of the results $(p<.05)$ indicated that the body region in which there were more injuries were the lower extremities (54.2\%), more specifically the hamstring muscles (23.7\%) and the most repeated diagnosis was the sprain (28.8\%). The most repeated type of injury was firs-time lesion (66.1\%), with impact (57.6\%) being the most frequent mode. Data were also obtained about the moment in which injuries occur, competition (61.0\%). 26.3\% suffer chronic pain due to a recurring injury. This study offers decisive information to specialists for the prevention of injuries in the preparation of elite players.

Key words: injury, beach handball, questionnaire, sand, performance.

\section{Introducción}

El balonmano playa es un deporte relativamente nuevo que comienza a popularizarse durante los últimos años de la década de los noventa (Sena \& Gomes, 2012). En sus orígenes, y hasta el día de hoy, se practica durante el periodo de temperaturas medias-altas, salvo excepciones como por ejemplo Brasil que lo practican durante todo el año. Con el tiempo, este deporte ha ido creciendo y tecnificándose, con campeonatos nacionales, europeos y mundiales organizados por las distintas federaciones, hablándose cada vez más de su posible inclusión como deporte olímpico (Zapardiel, 2018).

La distribución temporal del balonmano playa difiere de su versión en pista. Un partido se divide en dos tiempos de 10 minutos y 5 minutos de descanso, disputado por dos equipos de 4 jugadores cada uno, incluido el portero en defensa o especialista en ataque, y seis suplentes. Si al finalizar el tiempo reglamentario, ambos equipos tienen el mismo número de goles se llevará a cabo la «gol de oro» para desempatar (Karras, Chryssanthopoulos, Diafas, \& Science, 2007). Una de las características singulares, que lo hace más dinámico y espectacular que su análogo de pista, es la posibilidad de anotar un gol con puntación doble marcando un $360^{\circ} \mathrm{o}$ «giro» (si se realiza una rotación en el aire de $360^{\circ}$ previo al lanzamiento), si el gol es marcado por el portero o especialista mientras actúa como jugador de campo (no desde la zona de portería), si se consigue de penalti o si se marca en «fly», cuando el último pase se recepciona en el aire y se lanza a portería antes de caer (Bykova, Strelnykova, Pomeshchikova, \& Lyakhova, 2015; Morillo, 2009).

Como consecuencia de este dinamismo, la demanda fisiológica anaeróbica y aeróbica es muy alta (Miranda et al., 2014). Se producen numerosos desplazamientos y acciones de intensidad moderada a alta en los momentos técnicos más decisivos, distribuidos intermitentemente a lo largo del juego, donde la intensidad es menor (Pueo, JiménezOlmedo, Penichet-Tomás, Ortega Becerra, \& Espina Agulló, 2017). El elevado nivel de actividad anaeróbica se debe a que el balonmano playa está diseñado para que los contrataques ocurran en todo momento ya que cuando se anota un gol, el portero pone la pelota inmediatamente en juego(Silva et al., 2016).

Fecha recepción: 17-07-18. Fecha de aceptación: 07-01-19 Alfonso Penichet-Tomás

alfonso.penichet@gcloud.ua.es
Por otro lado, el terreno de juego también muestra diferencias con respecto al balonmano pista (Rules of the Game, 2010). Se desarrolla en un terreno de arena de 27 x 12 m con un área rectangular a 6 m de la portería. La superficie irregular arenosa tiende a deformarse ante la presión que ejercen los jugadores, lo que provoca una adaptabilidad constante al terreno y una producción de fuerzas de alta intensidad en músculos y articulaciones. Por ello, el terreno de juego de arena supone una gran carga para los esfuerzos necesarios en desplazamientos y saltos (Silva et al., 2016). Además, el juego se desarrolla en un ambiente con temperaturas relativamente altas $\left(>25^{\circ} \mathrm{C}\right)$ y elevados niveles de humedad (> 65\% ) (Karras et al., 2007), por lo que la posibilidad de sufrir una lesión aumenta si la preparación no ha sido la correcta. En consecuencia, las lesiones en balonmano playa afectan al aparato locomotor, como consecuencia de la interacción con un medio inestable (Hatzimanouil et al., 2017). Sin embargo, la dinámica de este deporte provoca otras lesiones no relacionadas con la superficie. Por ejemplo, aunque el contacto está prohibido y sancionado en este deporte, suelen producirse impactos contra el terreno de juego o choques violentos contra un oponente durante los momentos de ataque, derivando en posibles lesiones (Manavis, Hatsimanouil, Eleftherios, \& Bebetsos, 2008).

Las investigaciones sobre lesiones deportivas se utilizan principalmente para desarrollar hipótesis sobre sus causas de origen y así poder desarrollar intervenciones para prevenirlas (Galvez Aguilar, Campos Salazar, \& Chacon Araya, 2017; Giroto, Hespanhol Junior, Gomes, \& Lopes, 2017). El patrón lesivo en cada deporte puede diferir entre sus diferentes versiones y categorías (Manavis et al., 2008; Olmedilla Zafra, Andreu Alvarez, Ortín Montero, \& Blas Redondo, 2009; Souchon, Dosseville, Traclet, \& Coulomb-Cabagno, 2008). Por ejemplo, la incidencia lesiva en fútbol, voleibol o balonmano (Raya-González, Gómez Piqueras, \& Sánchez-Sánchez, 2018), entre otros, puede tener características tanto semejantes como diferentes respecto a fútbol playa (Shimakawa etal., 2016), vóley playa (Gea \& Molina, 2015) o balonmano playa (Hatzimanouil et al., 2017).

Actualmente, la literatura científica recoge numerosos estudios sobre lesiones en balonmano tanto en la categoría élite (Bere et al., 2015; Giroto et al., 2017; Møller et al., 2017) como en categorías de inferior nivel (Kim, 2012; Olsen, Myklebust, Engebretsen, \& Bahr, 2006; Souchon et al., 2008). Sin embargo, existe escasa literatura sobre lesiones en balonmano playa realizados sobre jugadores de élite. Desafortunadamente, no existe evidencia científica sobre qué lesiones son más 
comunes en deportistas de alto nivel español que practiquen este deporte como ocurre en otras disciplinas deportivas.

Por lo tanto, el presente estudio tiene como objetivo determinar cuáles son las regiones anatómicas y corporales que más lesiones han sufrido jugadores españoles de élite de balonmano playa y establecer el tipo, modo y momento más común en el que ocurren estas lesiones. Esta información es de gran utilidad para adecuar la preparación física y los programas de prevención con el propósito de poder reducir el número de lesiones y la posibilidad de aparición de dolores crónicos futuros.

\section{Método}

\section{Sujetos}

Ochenta deportistas españoles de élite de balonmano playa, procedentes de veinte diferentes clubs participantes en el Arena Handball Tour de la Real Federación Española de Balonmano (edad: $26.8 \pm 8.2$ años; experiencia: $6.4 \pm 5.0$ años; peso: $79.5 \pm 16.7$ kg.; estatura: 178.8 $\pm 8.7 \mathrm{~cm}$.), distribuidos en veintinueve porteros-especialista, catorce pivotes, trece alas derechas y veinticuatro alas izquierdas, participaron voluntariamente en el estudio. El 46\% de los sujetos (n = 37) habían sufrido una o más lesiones practicando balonmano playa mientras que el $54 \%$ restante $(n=43)$ nunca se había lesionado practicando este deporte. Todos los sujetos dieron su consentimiento por escrito antes de iniciarse el estudio, aprobado por el comité de ética de investigación de la Universidad deAlicante.

\section{Procedimiento}

Para la realización del estudio se utilizó un cuestionario adaptado (Penichet-Tomás et al., 2012) de uno previamente validado (Eloranta \& Tittonen, 2006). Los deportistas cumplimentaron el cuestionario con las lesiones sufridas practicando balonmano playa. Los sujetos especificaron la región anatómica de cada lesión (brazo, cadera, codo, columna, cuello, dedos mano, dedos pie, hombro, intercostal, isquiotibiales, muñeca, muslo, nariz, pie, pierna, tobillo u otro) para poder analizar también la incidencia lesiva según la región corporal: extremidades inferiores, superiores, tronco o cabeza. También indicaron el tipo de lesión, si se trataba de una nueva lesión, una lesión sufrida con anterioridad o una lesión incrementada. También especificaron el modo en el que sufrieron la lesión. Las lesiones por uso son las lesiones donde el deportista puede especificar la acción y el momento exacto en el que se produjo. Las lesiones por sobreuso son aquellas lesiones donde no se logra identificar un evento traumático desencadenante. Acontinuación, las lesiones por impacto son aquellas producidas por el choque de una superficie corporal contra un agente externo. Asimismo, se pidió a los deportistas que determinaran el momento en el que se produjo la lesión: calentamiento, entrenamiento o competición, y el diagnóstico de cada lesión (tendinitis, esguince, dislocación, rotura, factura, contusión, luxación, rotura muscular, fascitis, contractura, herida, fisura, microrrotura, sobrecarga, protrusión u otro).

En la última parte del cuestionario, se preguntó a los deportistas si sufrían dolor crónico en alguna región corporal y cuál era el motivo: una vieja lesión o debido a una mala preparación.

\section{Análisis estadístico}

Los datos fueron analizados con el programa informático Statistical Package Social Sciences (SPSS) v.22.0, para calcular los estadísticos descriptivos, las frecuencias y la comparación de porcentajes. Mediante el test Chi-cuadrado $\left(\mathrm{x}^{2}\right)$ para pruebas no paramétricas, se determinaron los niveles de significación estadística $p<.05$.

Figura 1

\section{Resultados}

La región anatómica que ha sufrido un mayor número de lesiones ha sido los músculos isquiotibiales con un 23.7\%, seguido del tobillo con un $13.6 \%$ y los dedos de la mano con un $10.3 \%$ (Figura 1). Los resultados expuestos en la Figura 2 indican que el diagnóstico que más se ha repetido en las lesiones es el esguince (28.8\%) seguido de la dislocación (10.2\%) y la fractura (10.2\%).

Después de agrupar las regiones anatómicas, según la parte del cuerpo en la que se encuentra, la Tabla 1 muestra que la región corporal donde más lesiones se han producido es en las extremidades inferiores con un 54.2\%. La segunda región corporal más castigada han sido las extremidades inferiores con un $25.4 \%$ seguido del tronco con un $16.9 \%$.

El tipo de lesión más repetida es la que ha ocurrido por primera vez con una incidencia del 66.1\%. Por otro lado, las lesiones reincidentes e incrementadas tan solo tuvieron una incidencia del 18.6\% y 15.3\% respectivamente. Las lesiones producidas a causa de un impacto son las más repetidas con un $57.6 \%$. Sin embargo, las lesiones por uso y uso excesivo, aquellas en las queno se logra identificar el momento traumático desencadenante, han ocurrido el $22.0 \%$ y $20.3 \%$, respectivamente.

Tras analizar el momento en el que se produjeron las lesiones, los resultados indican que el $61.0 \%$ de las lesiones ocurrieron en competición, mientras que sólo el 37.3\% de las lesiones fueron en los entrenamientos y el $1.7 \%$ durante el calentamiento.

La última parte del cuestionario hacía referencia al posible dolor crónico que pudieran sufrir los deportistas (Tabla 2). Los resultados muestran que el 28.7\% sufre dolor crónico. En concreto, el 26.3\% es debido a una vieja lesión frente al $2.5 \%$ que se debe a una mala preparación, es decir, lesiones producidas por una preparación específica inade-

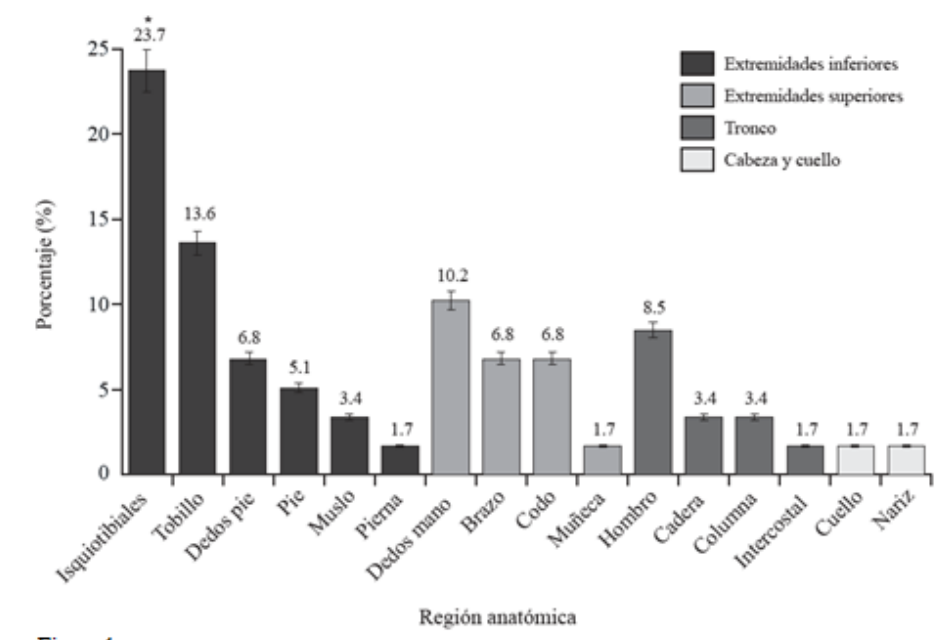

Porcentaje de lesiones por región anatómica ${ }^{*} p<.05$.

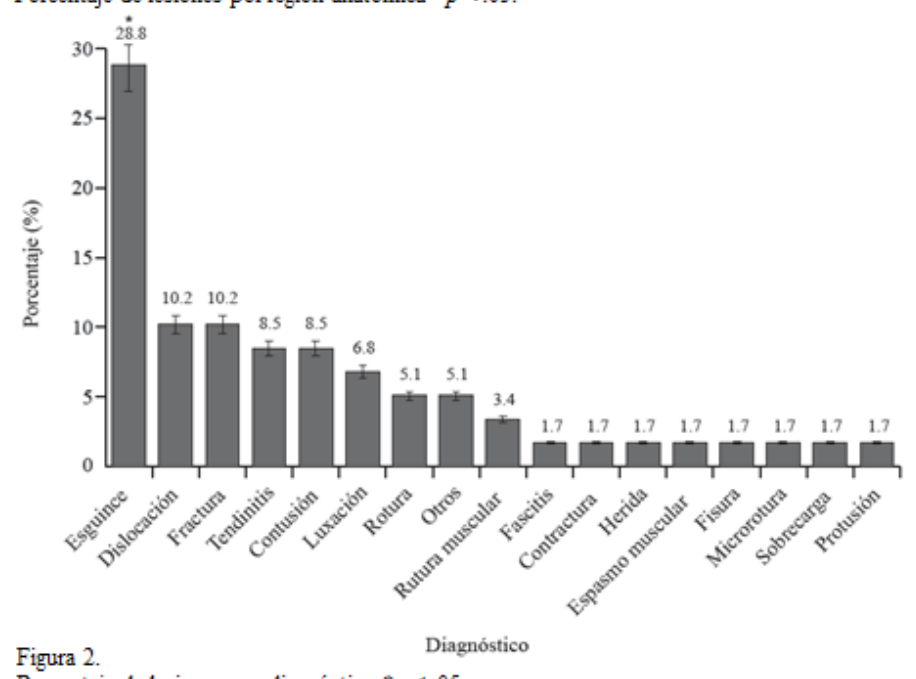

Porcentaje de lesiones por diagnóstico ${ }^{*} p<.05$. 
Tabla 1.

Características de las lesiones.

\begin{tabular}{llcc}
\hline & & Frecuencia (n=59) & Incidencia (\%) \\
\hline \multirow{4}{*}{ Región corporal } & Extremidades inferiores & 32 & $54.2^{*}$ \\
& Extremidades superiores & 15 & 25.4 \\
& Tronco & 10 & 16.9 \\
& Cabeza & 2 & 3.4 \\
\hline \multirow{2}{*}{ Tipo } & Nueva lesión & 39 & $66.1^{*}$ \\
& Reincidente & 11 & 18.6 \\
& Incrementada & 9 & 15.3 \\
\hline \multirow{2}{*}{ Modo } & Uso & 13 & 22.0 \\
& Uso excesivo & 12 & 20.3 \\
& Impacto & 34 & $57.6^{*}$ \\
\hline \multirow{2}{*}{ Momento } & Calentamiento & 1 & 1.7 \\
& Entrenamiento & 22 & 37.3 \\
${ }^{*} p<.05$. & Competición & 36 & $61.0^{*}$ \\
Tabla 2. & & & \\
& & &
\end{tabular}

Características de las lesiones crónicas.

\begin{tabular}{llcc}
\hline & & Frecuencia (n) & Incidencia (\%) \\
\hline \multirow{3}{*}{ Dolor crónico } & No & 57 & $71.3^{*}$ \\
& Sí́ & 23 & 28.7 \\
\cline { 2 - 4 } & Vieja lesión & 21 & $26.3^{*}$ \\
& Mala preparación & 2 & 2.5 \\
\hline \multirow{2}{*}{ Región corporal } & Extremidades inferiores & 12 & 15.0 \\
& Extremidades superiores & 6 & 7.5 \\
& Tronco & 5 & 6.3 \\
\hline${ }^{*}<05$
\end{tabular}

${ }^{* p}<.05$.

cuada. La región corporal donde más jugadores sufren dolor crónico son las extremidades inferiores con un $15 \%$, igual que ocurría anteriormente con la región corporal en la que más deportistas han sufrido algún tipo de lesión. Por otro lado, las extremidades superiores han tenido una incidencia de dolor crónico del 7.5\% y el tronco del 6.3\%.

Se han encontrado diferencias significativas $(\mathrm{p}<.05)$ en las frecuencias de lesiones de extremidades inferiores respecto al resto del cuerpo y de nuevas lesiones frente a reincidentes o incrementadas. Además, también se observan diferencias significativas en las lesiones producidas por impacto en lugar de por uso o uso excesivo, y en las ocurridas en competición frente a entrenamientos o calentamientos. Finalmente, las lesiones crónicas como causa de viejas lesiones han sido significativamente más frecuentes que las producidas por mala preparación.

\section{Discusión}

El balonmano playa es un deporte con un número muy bajo de lesiones tanto en competición como en entrenamientos. En el presente estudio el 54\% de los deportistas nunca han sufrido una lesión practicando este deporte. En el estudio realizado por Hatzimanouil et al. (2017) la tasa de incidencia de lesiones para hombres y mujeres fue de 12 y 11.36 lesiones respectivamente por cada mil horas de partido, y de 1.8 y 4 lesiones por cada mil horas de entrenamiento correspondiente. Por otro lado, tan sólo un $15.5 \%$ de los jugadores de balonmano playa sufrieron algún tipo de lesión en el estudio de Manavis et al. (2008).

El balonmano playa se practica sobre un terreno de arena seca, una superficie irregular ligeramente acolchada que supone una gran carga física para las extremidades inferiores. La región corporal más castigada por las lesiones del presente estudio fueron las extremidades inferiores (54.2\%), en concordancia con el último estudio realizado sobre lesiones de balonmano playa (Hatzimanouil et al., 2017) o el estudio realizado en los segundos Asian Beach Games (Al-Shaqsi, Al-Kashmiri,Al-Risi, \&Al-Mawali, 2012). Las extremidades superiores fue la segunda región corporal con mayor número de lesiones (25.4\%) como ocurrió con los deportistas de elite del estudio realizado por Hatzimanouil et al. (2017). Los pases y lanzamientos en balonmano playa se realizan con las extremidades superiores a gran velocidad debido al poco peso y pequeña dimensión del balón, y al gran nivel de fuerza de los jugadores (Sena \& Gomes, 2012). Las lesiones del hombro son comunes como consecuencia de la gran energía que se transmite durante el lanzamiento (Zapardiel, 2015). Además, existe una gran implicación de las extremidades superiores en blocajes y contragiros con una menor pero existente implicación del tren inferior. De la misma manera, el bloqueo es una acción en la que interviene en mayor medida el tren inferior. Todo ello explicaría la alta incidencia de lesiones de esta región corporal respecto a tronco, cabeza y cuello.
En estos desplazamientos y saltos, tanto ofensivos como defensivos, la fuerza de los músculos isquiotibiales es fundamental debido al ciclo estiramiento-acortamiento (CEA) (Koga et al., 2010; Palmer, Followay, \& Thompson, 2017). Los resultados mostraron como la región anatómica con mayor incidencia de lesión fueron los isquiotibiales (23.7\%) y el tobillo (13.6\%). Los jugadores de balonmano playa de élite (Hatzimanouil et al., 2017), así como los de futbol playa (Shimakawa et al., 2016), sufrieron más lesiones en la rodilla, seguido también del tobillo, como ocurre en el presente estudio. La irregularidad del terreno hace que los tobillos se vean sometidos a giros, flexiones laterales y longitudinales. La falta de protección en pies y tobillos aumenta el riesgo de lesiones. Este patrón se observa en otros deportes de playa, como el futbol playa, donde la mayor incidencia de lesión se encuentra en tales regiones anatómicas (Al-Shaqsi et al., 2012; Shimakawa et al., 2016).

El diagnóstico más repetido en las lesiones del presente estudio ha sido el esguince (28.8\%). Estos datos concuerdan con los resultados obtenidos en el estudio de Al-Shaqsi et al. (2012), realizado sobre catorce deportes de playa, donde el esguince tuvo la mayorincidencia(26.6\%), y donde el balonmano playa, tuvo como diagnóstico más repetido el esguince (30.3\%) y la contusión (30.3\%). Estos datos también coinciden con otros deportes de playa donde no hay contacto con el oponente como es el caso del vóley playa (Jimenez-Olmedo \& Penichet-Tomas, 2015). La contusión en el presente estudio tuvo una incidencia del 8.5\%, siendo el tercer diagnóstico más repetido, mientras que en el estudio realizado con jugadores de élite internacional de balonmano playa, tuvo una incidencia del $24.4 \%$ (Hatzimanouil et al., 2017). Este hecho puede ser debido a que los jugadores de balonmano playa de tan alto nivel tienen una mejor preparación que les protegerles de lesiones como los esguinces. Sin embargo, las posibles caídas y los posibles choques en los contragiros son más frecuentes en la élite aumentando las posibles lesiones por contusiones.

En relación al tipo de lesión, la nueva lesión fue la más repetida (66.1\%) y el impacto, definido como acción producida por el choque de una superficie corporal contra un agente externo tuvo mayor incidencia (57.5\%) frente a las lesiones por uso o uso excesivo. Las lesiones de los jugadores de élite internacional de balonmano playa (el $56.3 \%$ en hombres y el 69\% en mujeres) fueron producidas también por impacto (Hatzimanouil et al., 2017). Las lesiones en balonmano playa pueden producirse contra un adversario, jugador o portero, contra el terreno de juego o contra ambos. Estas acciones son comunes por la dinámica de este deporte, donde está permitido el choque entre jugadores que atacan y defienden en ciertos momentos del juego. Los impactos contra el terreno de juego suelen producirse en aquellas ocasiones que suponen un desequilibrio del jugador en ataque o defensa. Los impactos que producen contra jugador y terreno de juego, pueden producirse tras el intento de blocaje del defensor cuando el atacante intenta realizar el lanzamiento en giro sobre sí mismo. Los estudios concuerdan que las lesiones por impacto contra un adversario fueron las lesiones más repetidas con el 23.7\% (Al-Shaqsi et al., 2012), el 71.4\% (Manavis et al., 2008), y el $36.8 \%$ en hombres y el $43.2 \%$ en mujeres (Hatzimanouil et al., 2017).

El mayor número de lesiones ocurrieron en la competición(61.0\%) frente a entrenamientos o calentamientos. Estos datos concuerdan con los resultados obtenidos de las encuestas realizadas a los jugadores participantes en el Campeonato de Europa de 2007 (Manavis et al., 2008) donde el $57.1 \%$ de las lesiones se produjeron en competición. Igualmente, en los segundos Asian Beach Games, la mayoría de las lesiones ocurrieron en competición (75.4\%), un valor tres veces superior al de entrenamiento. Esto puede ser debido a que el nivel de entrenamiento y preparación física específica puede ser relativamente bajo respecto a la exigencia competitiva a la que posteriormente se someten, especialmente en deportes que se practican en periodos estivales. Según el estudio realizado sobre los Summer Olympic Games (2008), las lesiones que ocurrieron durante la competición fueron diferentes a las lesiones producidas durante los entrenamientos (Junge et al., 2009).

Las lesiones crónicas de jóvenes deportistas han sido objeto de estudio (Vetter \& Symonds, 2010) y uno de los factores que pueden 
incidirnegativamenteson las deficiencias técnicas (Henke, Luig, \& Schulz, 2014) y mala preparación o tratamiento. El presente estudio demostró que el $28.7 \%$ de los deportistas sufrían dolor crónico y la causa del 26.3\% de estos deportistas fue una vieja lesión. Las investigaciones futuras deberían estudiar también la etiología de las lesiones crónicas ya que también pueden afectar tanto a la salud como al rendimiento de los jugadores (Junge et al., 2009).

\section{Conclusiones}

Este estudio ha explorado el patrón lesivo en jugadores españoles de élite de balonmano playa. Sus resultados sugieren que el esguince ha sido la lesión con diagnóstico más repetido y la nueva lesión el tipo de lesión más repetida. El impacto fue el modo más repetido y la competición el momento en el que más lesiones se produjeron debido a la dinámica de juego que caracteriza este deporte.

El motivo por el cual la mayoría de jugadores sufrían dolor crónico fue una vieja lesión. Por tanto, no sólo es necesario un trabajo específico de prevención de lesiones para este perfil de jugadores, sino también adecuados programas de tratamiento que permitan una óptima recuperación.

En conclusión, este estudio determina que los jugadores de élite de balonmano playa sufren mayor número de lesiones en las extremidades inferiores, más concretamente en los músculos isquiotibiales. Estos datos ponen en énfasis la necesidad de programas específicos de prevención de lesiones para jugadores de élite de balonmano playa.

\section{Referencias}

Al-Shaqsi, S., Al-Kashmiri, A., Al-Risi, A., \& Al-Mawali, S. (2012). Sports injuries and illnesses during the second Asian Beach Games. British Journal of Sports Medicine, 46(11), 780-787.

Bere, T., Alonso, J.-M., Wangensteen, A., Bakken, A., Eirale, C., Dijkstra, H. P., ... Popovic, N. (2015). Injury and illness surveillance during the 24th Men's Handball World Championship 2015 in Qatar. British Journal of Sports Medicine, 49(17), 1151-1156.

Bykova, O., Strelnykova, Y., Pomeshchikova, I., \& Lyakhova, T. (2015). Changes of the level of high-speed and power abilities of beach handball players of 14-15 years old as a result of the specially selective exercises. Slobozhanskyi Herald of Science and Sport, 1(6), 26-29.

Eloranta, I., \& Tittonen, T. (2006). The reliability and validity of sport injury questionnaire in questions concerning acute and overused injuries. Physiotherapy, University of Jyväskylä.

Galvez Aguilar, W., Campos Salazar, C., \& Chacon Araya, Y. (2017). Epidemiology of injuries in students of Human Movement Sciences at the University of Costa Rica. Retos: Nuevas Tendencias En Educación Física, Deporte y Recreación, (31), 171-175.

Gea, G. M., \& Molina, J. J. (2015). Relationship between sport level competition and serving skill in female beach volleyball. Revista Internacional de Medicina y Ciencias de La Actividad Física y El Deporte, 15(59), 433-449.

Giroto, N., Hespanhol Junior, L. C., Gomes, M. R. C., \& Lopes, A. D. (2017). Incidence and risk factors of injuries in Brazilian elite handball players: A prospective cohort study. Scandinavian Journal of Medicine \& Science in Sports, 27(2), 195-202.

Hatzimanouil, D., Papasoulis, E., Terzidis, I., Kanioglou, A., Mavropoulou, A., \& Natsis, K. (2017). Injuries in elite athletes of beach handball. Journal of Human Sport and Exercise, 12(3), 689-697. https:// doi.org/10.14198/jhse.2017.123.13

Henke, T., Luig, P., \& Schulz, D. (2014). Sportunfälle im Vereinssport in Deutschland. Bundesgesundheitsblatt - Gesundheitsforschung Gesundheitsschutz, 57(6), 628-637.

Jimenez-Olmedo, J. M., \& Penichet-Tomas, A. (2015). Injuries and pathologies in beach volleyball players: A systematic review. Journal of Human Sport and Exercise, 10(4), 936-948. https://doi.org/ 10.14198/jhse.2015.104.09

Junge, A., Engebretsen, L., Mountjoy, M. L., Alonso, J. M., Renström, P. A. F. H., Aubry, M. J., \& Dvorak, J. (2009). Sports injuries during the Summer Olympic Games 2008. The American Journal of Sports Medicine, 37(11), 2165-2172.
Karras, D., Chryssanthopoulos, C., Diafas, V., \& Science, S. (2007). Body fluid loss during four consecutive beach handball matches in high humidity and environmental temperatures. Serbian Journal of Sports Sciences, 1(1), 15-20.

Kim, S. (2012). Sports injury and management depending upon participation level in female handball players. The Korean Society of Sports Science, 19(1), 997-1009.

Koga, H., Nakamae, A., Shima, Y., Iwasa, J., Myklebust, G., Engebretsen, L., ... Krosshaug, T. (2010). Mechanisms for noncontact anterior cruciate ligament injuries. The American Journal of Sports Medicine, 38(11), 2218-2225.

Manavis, K., Hatsimanouil, D., Eleftherios, S., \& Bebetsos, G. S. (2008). Injuries in beach handball. European Handball Federation Publications, 1-5.

Miranda, M., Toscano, L., Félix, G., Costa, D., Tavares, R., \& Marques, R. (2014). Food consumption by beach handball world-champion teams. Revista Brasileira de Ciências Da Saúde, 18(S4), 43-50.

Møller, M., Nielsen, R. O., Attermann, J., Wedderkopp, N., Lind, M., Sørensen, H., \& Myklebust, G. (2017). Handball load and shoulder injury rate: a 31-week cohort study of 679 elite youth handball players. British Journal of Sports Medicine, 51(4), 231-237.

Morillo, J. P. (2009). Balonmano Playa. Sevilla: Wanceulen.

Olmedilla Zafra, A., Andreu Alvarez, M. D., Ortín Montero, F. J., \& Blas Redondo, A. (2009). Injuries and young football players: an analysis in two clubs of Murcia Region. Retos: Nuevas Tendencias En Educación Física, Deporte y Recreación, 16, 63-66.

Olsen, O. E., Myklebust, G, Engebretsen, L., \& Bahr, R. (2006). Injury pattern in youth team handball: a comparison of two prospective registration methods. Scandinavian Journal of Medicine and Science in Sports, 16(6), 426-432.

Palmer, T., Followay, B., \& Thompson, B. (2017). Age-related effects on maximal and rapid hamstrings/quadriceps strength capacities and vertical jump power in young and older females. Aging Clinical and Experimental Research, 29(6), 1231-1239.

Penichet-Tomás, A., Jiménez-Olmedo, J. M., Saiz-Colomina, S., JoveTossi, M. A., Martínez-Carbonell, J. A., \& Silvestre-García, M. (2012). Incidence injury analysis on rowers in the spanish mediterranean fixed bench championship 2012. Journal of Human Sport and Exercise, 7(3), 648-657.

Pueo, B., Jiménez-Olmedo, J. M., Penichet-Tomás, A., Ortega Becerra, M., \& Espina Agulló, J. J. (2017). Analysis of time-motion and heart rate in elite male and female beach handball. Journal of Sports Science and Medicine, 16, 450-458.

Raya-González, J., Gómez Piqueras, P., \& Sánchez-Sánchez, J. (2018). Effect of an eccentric load resistance training program on the rehabilitation of medial collateral ligament injuries. A case study. Retos: Nuevas Tendencias En Educación Física, Deporte y Recreación, (33), 157-161.

Rules of the Game. (2010). Basilea: International Handball Federation.

Sena, J., \& Gomes, A. (2012). Dermatoglyph, somatotype and body composition in beach handball: Comparative study among different level of sportive qualification. Motricidade, 8(S2), 567-576.

Shimakawa, T., Shimakawa, Y., Kawasoe, Y., Yoshimura, K., Chinen, Y., Eimon, K., ... Ikeda, H. (2016). Beach soccer injuries during the Japanese National Championships. Orthopaedic Journal of Sports Medicine, 4(1), 232596711562563.

Silva, A. S., Coeli Seabra Marques, R., DE Azevedo Lago, S., Guedes Santos, D. A., Lacerda, L. M., Silva, D. C., \& Soares, Y. M. (2016). Physiological and nutritional profile of elite female beach handball players from Brazil. The Journal of Sports Medicine and Physical Fitness, 56(5), 503-509.

Souchon, N., Dosseville, F., Traclet, A., \& Coulomb-Cabagno, G. (2008). Sex of players and injuries in handball: Refereeing influence? Science \& Sports, 23, 32-34.

Vetter, R. E., \& Symonds, M. L. (2010). Correlations between injury, training intensity, and physical and mental exhaustion among college athletes. Journal of Strength and Conditioning Research, 24(3), 587-596.

Zapardiel, J. C. (2015). Valoración isocinética de los músculos rotadores del complejo articular del hombro en jugadores de balonmano playa. Universidad de Alcalá.

Zapardiel, J. C. (2018). Beach handball European championships analysis Zagreb 2017. EHF Web Periodical, (January), 1-27. 